\title{
Durvalumab Plus Tremelimumab in Solid Tumors: A Systematic Review
}

\author{
Caterina Arru • Maria Rosaria De Miglio (D) - Antonio Cossu • \\ Maria Rosaria Muroni · Ciriaco Carru • Angelo Zinellu • \\ Panagiotis Paliogiannis
}

Received: April 1, 2021 / Accepted: May 15, 2021 / Published online: June 8, 2021

(C) The Author(s) 2021

\begin{abstract}
Introduction: Cancer immunotherapy represents one of the most important innovations in modern medicine. Durvalumab is an anti-programmed cell death ligand 1 (PDL-1) agent which is currently under investigation in several studies in combination with the anti-cytotoxic T lymphocyte-associated protein 4 (CTLA4) drug tremelimumab. The aim of this review was to systematically identify and revise the current scientific literature investigating the combination of these two drugs in solid tumors. Methods: A digital search on the Medline (PubMed interface) and Scopus databases for
\end{abstract}

Caterina Arru and Maria Rosaria De Miglio contributed equally to this work.

Supplementary Information The online version contains supplementary material available at https:// doi.org/10.1007/s12325-021-01796-6.

C. Arru - C. Carru - A. Zinellu · P. Paliogiannis Department of Biomedical Sciences, University of Sassari, Viale San Pietro 43, 07100 Sassari, Italy

M. R. De Miglio $(\bowtie) \cdot$ A. Cossu · M. R. Muroni Department of Medical, Clinical and Experimental Sciences, University of Sassari, Viale San Pietro 43, 07100 Sassari, Italy

e-mail: demiglio@uniss.it

C. Carru · P. Paliogiannis

Laboratory Quality Control Unit, University

Hospital Sassari (AOU-SS), 07100 Sassari, Italy articles published from inception to 26 February 2021 was performed. The terms used for the search were durvalumab AND tremelimumab. Trials reported in English involving adult patients with solid cancers treated with the combination durvalumab plus tremelimumab were retrieved; the references of the articles were cross-checked to identify missing papers.

Results: The electronic search produced 267 results; after exclusion of duplicates, irrelevant articles, reviews, and papers not in English or missing data, 19 articles were included for revision. The total number of patients treated with the combination of durvalumab and tremelimumab in the studies retrieved was 2052.

Conclusion: The combination of durvalumab plus tremelimumab showed some oncological advantages in comparison with traditional chemotherapies in some subsets of tumors, but generally has not shown consistent advantages in comparison with the employment of durvalumab monotherapy. A number of the studies examined had intrinsic methodological limitations; therefore, future well-designed studies involving larger cohorts are warranted.

Keywords: CTLA-4; Immunotherapy; PDL-1; Solid tumor; Tremelimumab 


\section{Key Summary Points}

Immunotherapy represents one of the most important innovations in modern clinical oncology, and numerous solid tumors are currently treated with immune checkpoint inhibitors.

Durvalumab is an anti-PDL-1 agent which was introduced in clinical practice in 2017, while tremelimumab is an antiCTLA-4 drug under investigation in several settings.

The combination of durvalumab plus tremelimumab has been recently investigated in thoracic, gastrointestinal, genitourinary, and other tumors.

We performed a systematic review of the literature and identified 19 articles reporting results on the combination of durvalumab plus tremelimumab.

Globally, the combination of these two drugs showed only sporadic benefits in comparison with the use of durvalumab alone or other treatments compared in the trials reviewed.

\section{DIGITAL FEATURES}

This article is published with digital features, including a summary slide, to facilitate understanding of the article. To view digital features for this article go to https://doi.org/10.6084/ m9.figshare.14595981.

\section{INTRODUCTION}

Cancer immunotherapy represents one of the most important innovations in modern medicine. Despite the involvement of immunity against cancer and the concept of neoplastic immunosurveillance dating back to the second half of the twentieth century, the modern era of immunotherapy started substantially in the last two decades with the discovery of cytotoxic T lymphocyte-associated protein 4 (CTLA-4)blocking antibodies, which can enhance antitumor immunity $[1,2]$. Ipilimumab and tremelimumab were the first anti-CTLA-4 human antibodies which entered clinical trials in patients with advanced cancer; ipilimumab was approved by the US Food and Drug Administration (FDA) in 2011 for the treatment of patients with metastatic melanoma [3]. Further drugs were subsequently investigated with the discovery of another pivotal immune checkpoint, the programmed cell death receptor 1 (PD-1) protein, which negatively regulates antitumor T cell function when bound to programmed cell death ligand 1 (PDL-1) [4]. Pembrolizumab and nivolumab were the first PD-1blocking antibodies approved by the FDA for the treatment of melanoma in 2014 and advanced non-small cell lung cancer (NSCLC) in 2015 , respectively $[5,6]$. Since then, numerous other medications have been introduced in clinical practice, and are currently used alone or integrated in combinations with other therapies (surgery, traditional chemotherapy, radiation therapy, etc.) in first-line or subsequent treatment protocols for the management of several solid tumors.

Durvalumab is a human IgG monoclonal antibody directed at the PDL-1 molecule. This molecule is expressed on the cell membrane of dendritic cells, macrophages, T cells, as well as in cancer cells. In the last case, upon interaction with PD1, PDL-1 causes inhibition of T cell activation and a reduced immunologic response against cancer cells [7]. Durvalumab, which blocks PDL-1 and its inhibitory effects on T cells, was initially approved by the FDA in 2017 for the treatment of locally advanced or metastatic urothelial cancer, as well as stage III unresectable NSCLC [8], and it is currently under investigation in numerous other solid tumors, alone or in combination with other treatments. Often, durvalumab is tested in combination with other immunotherapy agents which target CTLA-4, in order to enhance the spectrum of their immunological action, the therapeutic effect, and the oncological 
outcomes, possibly reducing side and undesirable effects $[8,9]$.

Most of the studies recently designed to investigate durvalumab-based combination therapies combine durvalumab with tremelimumab, an anti-CTLA- 4 antibody that has been tested in clinical trials for melanoma, colon cancer, gastric cancer, and mesothelioma [10-12]. Tremelimumab alone in these tumors globally showed low antineoplastic activity and considerable (although treatable) side effects [13]. Its performance in combination with other drugs is currently under investigation in trials including several types of solid tumors. The aim of the present review is to systematically identify studies publishing oncological outcomes regarding the combination of durvalumab plus tremelimumab in solid cancers, and to discuss their results and conclusions, along with the perspectives for future studies.

\section{MATERIALS AND METHODS}

An electronic search on the Medline (PubMed interface) and Scopus databases for articles published from inception to 26 February 2021 was performed. The terms used for the search were durvalumab AND tremelimumab. The inclusion criteria were (a) full-text articles reporting on series of adult patients with solid tumors treated with durvalumab and tremelimumab combined, regardless of their design; (b) English language; (c) reports with data regarding oncological outcomes and side effects available; (d) studies approved by an ethics committee and performed in accordance with the principles of the declaration of Helsinki. Abstracts were screened, and the selected fulltext articles were independently reviewed by two investigators (Caterina Arru and Panagiotis Paliogiannis); in cases of discordance, a third investigator was involved to establish the relevance of the papers discussed (Maria Rosaria De Miglio). References of the retrieved articles were also cross-checked to identify missing studies. This article is based on previously conducted studies and does not contain any new studies with human participants or animals performed by any of the authors.

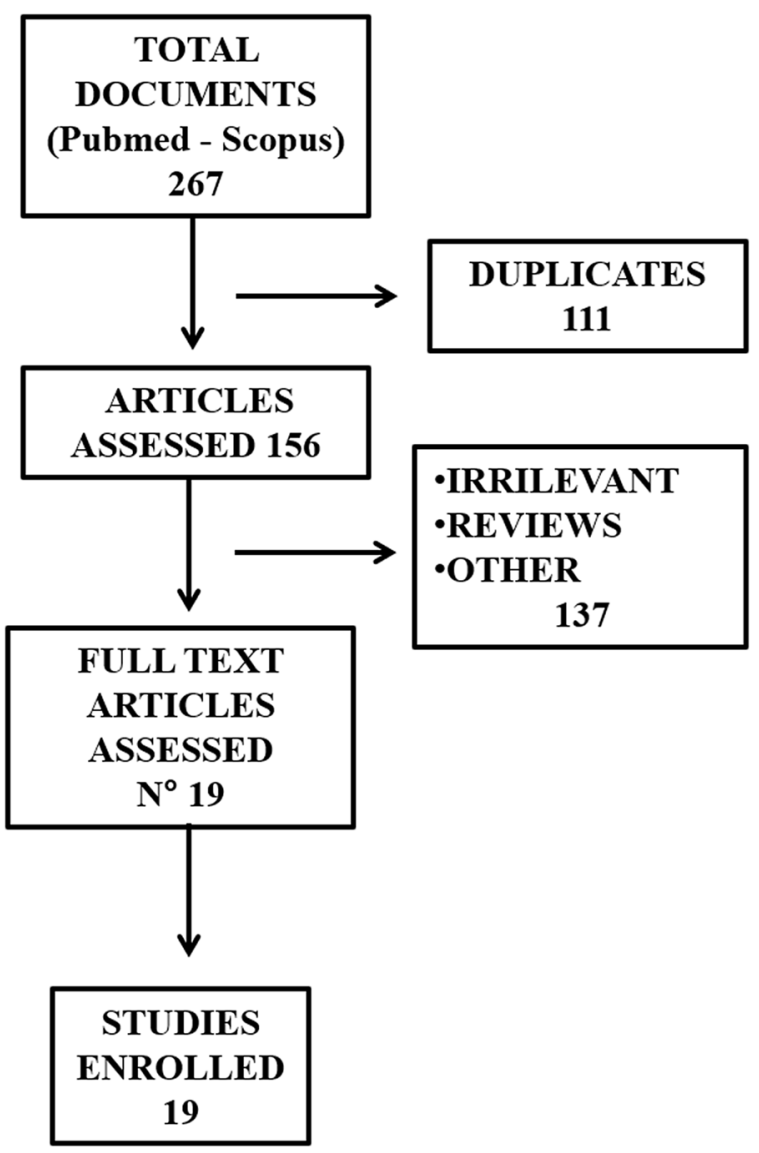

Fig. 1 Flowchart depicting the literature search strategy and results

\section{RESULTS}

The electronic search identified 267 articles; after exclusion of duplicates, irrelevant articles, reviews, and papers not in English or with missing data, 19 articles were finally included for revision [14-32]. Figure 1 depicts a flowchart describing the details of the document screening process, while Table 1 reports the principal details of the articles finally selected. The total number of patients treated with the combination of durvalumab and tremelimumab in the studies enrolled was 2052. Seven studies reported on patients affected by lung cancer and mesothelioma, two affected by head and neck tumors, five by gastrointestinal cancers, four by urogenital malignancies, while one article investigated multiple types of solid malignancies. The oncological outcomes of 
Table 1 Main characteristics of the articles selected and revised

\begin{tabular}{|c|c|c|c|c|}
\hline $\begin{array}{l}\text { Authors, country, } \\
\text { year }\end{array}$ & Cancer type & Treatments compared & $\begin{array}{l}\text { Patients treated with } \\
\text { Durv + Trem }\end{array}$ & References \\
\hline \multicolumn{5}{|l|}{ Lung and mesothelioma } \\
\hline $\begin{array}{l}\text { Antonia et al.; USA, } \\
2016\end{array}$ & NSCLC & Durv + Trem & 102 & {$[14]$} \\
\hline $\begin{array}{l}\text { Rizvi et al.; } \\
\text { multinational, } 2020\end{array}$ & NSCLC & Durv vs Durv + Trem vs C & 371 & {$[15]$} \\
\hline $\begin{array}{l}\text { Planchard et al.; } \\
\text { multinational, } 2020\end{array}$ & NSCLC & $\begin{array}{l}\text { Durv vs Durv }+ \text { Trem vs Trem vs } \\
\text { SoC }\end{array}$ & 174 & {$[16]$} \\
\hline $\begin{array}{l}\text { Juergens et al.; } \\
\text { Canada, } 2020\end{array}$ & NSCLC & Durv + Trem + several C & 73 & {$[17]$} \\
\hline $\begin{array}{l}\text { Goldman et al.; } \\
\text { multinational, } 2020\end{array}$ & SCLC & $\begin{array}{l}\text { Durv }+ \text { Trem }+ \text { Plat-Eto vs } \\
\text { Durv }+ \text { Plat-Eto vs Plat-Eto }\end{array}$ & 268 & {$[18]$} \\
\hline $\begin{array}{l}\text { Pakkala et al.; USA, } \\
2020\end{array}$ & SCLC & $\begin{array}{l}\text { Durv }+ \text { Trem }+ \text { Radio vs } \\
\text { Durv }+ \text { Trem }\end{array}$ & 18 & [19] \\
\hline $\begin{array}{l}\text { Juergens et al.; } \\
\text { Canada, } 2020\end{array}$ & SCLC & Durv + Trem + several C & 18 & {$[17]$} \\
\hline $\begin{array}{l}\text { Calabrò et al.; Italy, } \\
2018\end{array}$ & Mesothelioma & Durv + Trem & 40 & {$[20]$} \\
\hline $\begin{array}{r}\text { Juergens et al.; } \\
\text { Canada, } 2020\end{array}$ & Mesothelioma & Durv + Trem + several C & 7 & {$[17]$} \\
\hline $\begin{array}{l}\text { Kim et al.; Korea, } \\
2020\end{array}$ & $\begin{array}{l}\text { Pulmonary } \\
\text { sarcomatoid } \\
\text { carcinoma }\end{array}$ & Durv + Trem & 18 & {$[21]$} \\
\hline \multicolumn{5}{|l|}{ Head and neck cancer } \\
\hline $\begin{array}{l}\text { Siu et al.; } \\
\text { multinational, } 2018\end{array}$ & HNSCC & Durv vs Trem vs Durv + Trem & 133 & {$[22]$} \\
\hline $\begin{array}{l}\text { Ferris et al.; } \\
\text { multinational, } 2020\end{array}$ & HNSCC & Durv vs Durv + Trem vs SoC & 247 & {$[23]$} \\
\hline $\begin{array}{l}\text { Juergens et al.; } \\
\text { Canada, } 2020\end{array}$ & HNSCC & Durv + Trem + several C & 1 & {$[17]$} \\
\hline \multicolumn{5}{|l|}{ Gastrointestinal cancer } \\
\hline $\begin{array}{l}\text { Segal et al.; USA, } \\
2021\end{array}$ & Colorectal cancer & Durv + Trem + Radio & 24 & {$[24]$} \\
\hline $\begin{array}{l}\text { Kelly et al.; } \\
\text { multinational, } 2020\end{array}$ & $\begin{array}{l}\text { Gastric and } \\
\text { gastroesophageal } \\
\text { cancer }\end{array}$ & Durv vs Trem vs Durv + Trem & 52 & {$[25]$} \\
\hline
\end{tabular}


Table 1 continued

\begin{tabular}{|c|c|c|c|c|}
\hline $\begin{array}{l}\text { Authors, country, } \\
\text { year }\end{array}$ & Cancer type & Treatments compared & $\begin{array}{l}\text { Patients treated with } \\
\text { Durv + Trem }\end{array}$ & References \\
\hline $\begin{array}{l}\text { O'Reilly et al.; USA, } \\
2019\end{array}$ & Pancreatic cancer & Durv vs Durv + Trem & 32 & {$[26]$} \\
\hline Xie et al.; USA, 2020 & Pancreatic cancer & $\begin{array}{l}\text { Durv }+ \text { Radio vs } \\
\text { Durv }+ \text { Trem }+ \text { Radio }\end{array}$ & 35 & [27] \\
\hline $\begin{array}{r}\text { Juergens et al.; } \\
\text { Canada, } 2020\end{array}$ & Pancreatic cancer & Durv + Trem + several C & 4 & {$[17]$} \\
\hline $\begin{array}{l}\text { Boilève et al.; France, } \\
2021\end{array}$ & Biliary tract cancer & $\begin{array}{l}\text { Durv }+ \text { Trem vs } \\
\text { Durv }+ \text { Trem }+ \text { paclitaxel }\end{array}$ & 20 & {$[28]$} \\
\hline \multicolumn{5}{|l|}{ Urogenital cancer } \\
\hline $\begin{array}{l}\text { Gao et al.; USA, } \\
2020\end{array}$ & Urothelial cancer & Durv + Trem & 45 & [29] \\
\hline $\begin{array}{l}\text { Powels et al.; } \\
\text { multinational, } 2020\end{array}$ & Urothelial cancer & Durv vs Durv + Trem + SoC & 340 & {$[30]$} \\
\hline $\begin{array}{l}\text { Sarfaty et al.; USA, } \\
2021\end{array}$ & $\begin{array}{l}\text { Non-urothelial } \\
\text { urinary cancer }\end{array}$ & Durv + Trem & 13 & {$[31]$} \\
\hline $\begin{array}{r}\text { Juergens et al.; } \\
\text { Canada, } 2020\end{array}$ & Bladder cancer & Durv + Trem + several C & 9 & {$[17]$} \\
\hline $\begin{array}{l}\text { Necchi et al.; Italy, } \\
2018\end{array}$ & Germ cell tumors & Durv vs Durv + Trem & 11 & {$[32]$} \\
\hline
\end{tabular}

$C$ chemotherapy, Durv durvalumab, HNSCC head and neck squamous cell cancer, NSCLC non-small cell lung cancer, PlatEto platinum-etoposide, Radio radiation therapy, $S C L C$ small cell lung cancer, $S o C$ standard of care, Trem tremelimumab

patients treated with durvalumab plus tremelimumab were variably compared within several patient groups, i.e., receiving traditional chemotherapy, radiation therapy, durvalumab alone, tremelimumab alone, etc. The high heterogeneity of cases and controls, along with the small number of patients, prevented a metaanalysis; therefore, a systematic revision and discussion of the articles found has been performed. The PRISMA guidelines were used to report methods and results, and the related checklist is available as supplementary material.

\section{DISCUSSION}

\section{Durvalumab Plus Tremelimumab in Lung Cancer and Malignant Mesothelioma}

Lung cancer is one of the most common and lethal cancers with more than 2,200,000 new cases and approximately 1,800,000 deaths estimated worldwide in 2020 [33]. NSCLC accounts for approximately $80-85 \%$ of lung cancers comprising the most common histotypes like adenocarcinoma, squamous cell carcinoma, and large cell carcinoma, while small cell lung cancer (SCLC), which accounts for the remaining $10-15 \%$, is generally treated with chemo- and radiotherapy with high relapse and mortality 
rates [34, 35]. Targeted therapies and immunotherapy with immune checkpoint inhibitors (ICIs) targeting PD-1 or its ligand PDL-1 as monotherapies or in combination with anti-CTLA-4 drugs reshaped the landscape of the treatment of patients with metastatic NSCLC and represent a great opportunity for the treatment of SCLC $[35,36]$.

Focusing on NSCLC, nivolumab, pembrolizumab, atezolizumab, and cemipimab, alone or in combination with platin-based or other chemotherapies, are currently established weapons in the armamentarium of clinical oncologists for first-line treatment of squamous and non-squamous tumors. Durvalumab has been used since 2017 to treat patients with unresectable stage III NSCLC whose disease has not progressed after platinum-based chemoand radiation therapy [37, 38]. It has been hypothesized that the combination with tremelimumab may amplify anti-tumor $\mathrm{T}$ cell responses through immune checkpoint blockade and provide additive or synergistic activity, as combination therapies of the two antibodies have shown clinical activity in patients with advanced NSCLC in phase I and II investigations [39]. The first clinical study performed to evaluate such a hypothesis and the safety of the scheme was performed by Antonia et al. in 2016 [14]; the authors evaluated durvalumab plus tremelimumab in 102 immunotherapy-naïve patients with advanced NSCLC in a non-randomized study at five cancer centers in the USA. Over approximately 2 years, treatment-related grade 3 and 4 adverse events were observed in $36 \%$ of the cases, and among the 22 dead patients observed during the study, three were shown to have died because of the treatment. The authors concluded that durvalumab plus tremelimumab combination showed a manageable tolerability profile; interestingly, clinical activity was noted regardless of PDL-1 expression.

Our search identified two better designed trials, which were published in 2020 (Table 2). The randomized phase III MYSTIC clinical trial investigated whether first-line treatment with durvalumab, with or without tremelimumab, improved survival in patients with metastatic NSCLC [15]. The study included 1118 patients and was conducted for more than 3 years at 203 cancer centers in 17 countries. Patients received treatment with durvalumab alone, durvalumab plus tremelimumab, or chemotherapy. In this study, first-line treatment with durvalumab did not significantly improve overall survival (OS) compared with chemotherapy, but it was associated with a lower risk of death, suggesting a long-term advantage. Although statistical significance was not reached, the MYSTIC study evidenced that first-line treatment with durvalumab was associated with greater OS compared with patients treated with chemotherapy alone, whereas durvalumab plus tremelimumab did not significantly improve OS or progression-free survival (PFS) compared with chemotherapy.

The ARCTIC trial, a phase III, randomized, open-label study, evaluated durvalumab with or without tremelimumab versus standard of care (SoC) as a third-line treatment [16]. The study included patients from 205 centers in 26 countries for approximately 18 months. The trial was designed to include two independent studies: a first one (study A) with 126 patients with PDL-1 TC $\geq 25 \%$ treated with durvalumab or SoC, and a second study (study B) with 469 patients with PDL-1 TC $<25 \%$ treated with durvalumab plus tremelimumab, SoC, durvalumab alone, or tremelimumab alone. In study A, the median OS was 11.7 (95\% confidence interval (CI) 8.2-17.4) months with durvalumab versus 6.8 (95\% CI 4.9-10.2) months with SoC, while the median PFS was 3.8 (95\% CI 1.9-5.6) months with durvalumab versus 2.2 (95\% CI 1.9-3.7) months for those treated with SoC. In study B, the median OS was 11.5 (95\% CI 8.7-14.1) months with the combination durvalumab plus tremelimumab, and 8.7 (95\% CI 6.5-11.7) months in those treated with SoC, while the median PFS was 3.5 (95\% CI 2.3-4.6 / 1.9-3.9) months in both groups. Adverse events occurred most often in SoC than in durvalumab monotherapy or durvalumab plus tremelimumab treatment. The authors concluded that durvalumab alone showed significantly improved OS and PFS compared with SoC, especially in patients with PDL-1 $\geq 25 \%$; similarly, the OS and PFS of durvalumab plus tremelimumab were higher when compared with SoC in patients with PDL-1 TC $<25 \%$. The 
Table 2 Oncological outcomes of the combination of durvalumab plus tremelimumab in comparison with other treatments in patients affected by lung cancers or malignant mesothelioma

\begin{tabular}{|c|c|c|c|c|c|c|}
\hline $\begin{array}{l}\text { Author } \\
\text { [References] }\end{array}$ & $\begin{array}{l}\text { Value }(95 \% \\
\text { CI) }\end{array}$ & \multicolumn{4}{|l|}{ Treatments } & Comment \\
\hline & & $\mathrm{D}+\mathrm{T}$ & & & & \\
\hline \multirow[t]{3}{*}{ Antonia et al. [14] } & $\mathrm{AE}$ & $36 \%$ & & & & Single-arm study \\
\hline & & & & & & Immunotherapy-naïve patients \\
\hline & & $\mathrm{D}$ & $\mathrm{D}+\mathrm{T}$ & $\mathrm{C}$ & & \\
\hline \multirow[t]{5}{*}{ Rizvi et al. [15] } & OS m & 16.3 & 11.9 & 12.9 & & MYSTIC trial \\
\hline & PFS m & 4.7 & 3.9 & 5.4 & & First-line treatment \\
\hline & ORR \% & $35.60 \%$ & $34.40 \%$ & $37.70 \%$ & & \\
\hline & & Study A & & Study B & & \\
\hline & & $\mathrm{D}$ & SoC & $\mathrm{D}+\mathrm{T}$ & SoC & \\
\hline \multirow[t]{5}{*}{ Planchard et al. [17] } & OS m & 11.7 & 6.8 & 11.5 & 8.7 & ARCTIC trial \\
\hline & PFS m & 3.8 & 2.2 & 3.5 & 3.5 & Third-line treatment \\
\hline & $\mathrm{AE}$ & $9.70 \%$ & $44.40 \%$ & $22.00 \%$ & $36.40 \%$ & Study A: PDL-1 TC $\geq 25 \%$ \\
\hline & & & & & & Study B: PDL-1 TC < 25\% \\
\hline & & $\mathrm{D}+\mathrm{T}+\mathrm{PE}$ & $\mathrm{D}+\mathrm{PE}$ & $\mathrm{PE}$ & & \\
\hline \multirow[t]{3}{*}{ Goldman et al. [18] } & OS $\mathrm{m}$ & 10.4 & 12.9 & 10.5 & & CASPIAN trial \\
\hline & PFS m & 4.9 & 5.1 & 5.4 & & First-line treatment \\
\hline & & $\mathrm{D}+\mathrm{T}$ & & SBRT & & \\
\hline \multirow[t]{4}{*}{ Pakkala et al. [19] } & ORR & $0 \%$ & & $28.60 \%$ & & More than 2 previous chemotherapy \\
\hline & PFS m & 2.1 & & 3.3 & & lines \\
\hline & OS $\mathrm{m}$ & 2.8 & & 5.7 & & \\
\hline & & $\mathrm{D}+\mathrm{T}$ & & & & \\
\hline \multirow[t]{4}{*}{ Calabrò et al. [20] } & PFS m & 5.7 & & & & NIBIT-MESO-1 trial \\
\hline & OS $\mathrm{m}$ & 16.6 & & & & Single-arm study \\
\hline & $\mathrm{AE}$ & $75 \%$ & & & & First-line treatment \\
\hline & & $\mathrm{D}+\mathrm{T}$ & & & & \\
\hline \multirow[t]{2}{*}{ Kim et al. [21] } & PFS m & 5.9 & & & & Single-arm study \\
\hline & OS m & 15.4 & & & & First-line treatment \\
\hline
\end{tabular}

$A E$ treatment-related adverse events, $C$ chemotherapy, $C I$ confidence interval, $D$ durvalumab, ORR objective response rate, $O S m$ median overall survival, $P D L-1 T C$ tumor cell programmed cell death ligand 1, PE platinum-etoposide, PFS $m$ median progression-free survival, $S B R T$ stereotactic body radiation therapy, $S o C$ standard of care, $T$ tremelimumab 
authors underlined the need for an accurate classification of the patients in terms of PDL-1 positivity, but globally did not show any substantial advantage of the combination durvalumab plus tremelimumab in comparison to durvalumab alone.

A group of 73 patients with NSCLC treated with durvalumab plus tremelimumab has been also included in the multicenter phase Ib Canadian Cancer Trials Group study published in 2020 [17]. The study sought to establish a recommended dose for durvalumab with or without tremelimumab in combination with standard platinum-doublet chemotherapy in patients who had histologically and/or cytologically confirmed advanced, metastatic, recurrent, or unresectable cancer for which there were no curative treatment options [17]. The objective response rate (ORR) was 51\% (95\% CI 39-63\%), with a median duration of 6.8 (95\% CI 4.2-9.9) months, while the median PFS was 6.5 (95\% CI 5.5-9.4) months, and the median OS was 19.8 (95\% CI 14.8-not yet reached) months. The efficacy among patients with NSCLC was therefore encouraging; this study showed also better responses in PDL-1positive subtypes.

Globally, the studies performed on NSCLC did not show any substantial advantage of the combination of durvalumab plus tremelimumab among durvalumab alone, despite the better outcomes in comparison with chemotherapy. PDL-1 classification of the tumors appears to be particularly interesting in order to identify subgroups of better responders; further trials are necessary to better clarify this finding.

Regarding SCLC, it is well known that approximately two-thirds of the patients are diagnosed with advanced stage disease, and that the prognosis for these patients is particularly poor, with a 2-year survival rate of less than 5\% when treated with platinum-doublet chemotherapy [40]. Immunotherapy represents an extremely attractive perspective in this setting, and several trials are currently ongoing. The CASPIAN trial is a phase III randomized controlled trial involving 209 cancer centers in 23 countries worldwide, with 805 patients enrolled over 1 year and randomly assigned to treatment with durvalumab plus tremelimumab plus platinum-etoposide $(n=268)$ versus durvalumab plus platinum-etoposide $(n=268)$, or platinum-etoposide alone $(n=269)$ [18]. The combination durvalumab plus tremelimumab with platinum-etoposide did not show a significant improvement in OS compared with platinum-etoposide alone; in fact, median OS was 10.4 (95\% CI 9.6-12.0) months compared with 10.5 (95\% CI 9.3-11.2) months. In contrast, durvalumab plus platinum-etoposide showed a sustained improvement in OS compared with platinum-etoposide, with a median OS of 12.9 (95\% CI 11.3-14.7) months. The authors suggest that durvalumab plus platinumetoposide should be the new standard of care for first-line treatment of extended stage SCLC.

Pakkala et al. performed a study with the aim to evaluate the efficacy of ICIs with or without radiation in patients with SCLC [19]. In particular, the primary objective of this phase II study was to determine the efficacy of the combination of durvalumab and tremelimumab with or without stereotactic body radiation therapy (SBRT) in relapsed SCLC, using as primary endpoints the PFS and ORR. The study enrolled a total of 18 patients who were given either durvalumab and tremelimumab without SBRT (arm A, nine patients) or SBRT to a selected tumor site followed by durvalumab plus tremelimumab (arm B, nine Patients). The median PFS and ORR were 2.1 (95\% CI 0.8-3.2) months and $0 \%$ in arm $\mathrm{A}$, and 3.3 (95\% CI $0.9-4.9)$ months and $28.6 \%$ in arm B. Median OS was 2.8 (95\% CI 0.8-12.4) months in arm A and 5.7 (95\% CI 1.6-14.5) months in arm B. The study showed that the combination of the drugs with and without SBRT was safe, but did not show a significant efficacy in relapsed SCLC. In other words, also in SCLC the combination of durvalumab with tremelimumab did not show any notable advantage, even when combined with radiation therapy. Obviously, these findings need confirmation in further studies.

To date, only sporadic studies have investigated the role of durvalumab plus tremelimumab in rarer thoracic tumors. Calabrò et al. performed recently a study (NIBIT-MESO-1) on malignant mesothelioma, an aggressive tumor originating from mesothelial surfaces, 
characterized by extremely poor prognosis, and generally managed with a multidisciplinary approach; nivolumab is currently the only ICI recommended for first-line treatment of patients with malignant pleural mesothelioma, while pembrolizumab and ipilimumab (in combination with nivolumab) are employed in subsequent therapy regiments [41-43]. NIBITMESO-1 was an open-label, non-randomized phase 2 study performed in Italy with the aim to evaluate the efficacy and safety of first- or second-line durvalumab plus tremelimumab in patients with malignant mesothelioma [20]. The study enrolled 40 patients with unresectable pleural or peritoneal mesothelioma. Patients were followed up for a median of 19.2 (interquartile range (IQR) 13.8-20.5) months, and the median immune-related PFS was 8.0 (95\% CI 6.7-9.3) months, while the median PFS was 5.7 (95\% CI 1.7-9.7) months, and the median OS was $16.6 \quad(95 \%$ CI 13.1-20.1) months. Seventy-five percent of the patients experienced treatment-related adverse events, but toxicity was generally manageable and reversible. Therefore, the combination of the drugs appeared attractive in this study, with a good safety profile.

Pulmonary sarcomatoid carcinoma (PSC) is a rare type of lung cancer (less than $1 \%$ of all lung cancers) $[44,45]$, which is highly resistant to conventional cytotoxic chemotherapy [46]. The clinical course is aggressive with a poor prognosis and a median OS of approximately 6 months, even in the early stages of the disease [46-48]. Therefore, new therapeutic strategies that can improve life expectancy of patients with PSC are urgently needed. The KCSG-LU1607 study investigated the efficacy and safety of durvalumab and tremelimumab combined for the treatment of recurrent or metastatic PSCs [21]. This non-randomized, open-label phase II study included 18 patients and showed a median PFS and OS of 5.9 (95\% CI 1.1-11.9) months and 15.4 (95\% CI 11.1-not reached) months, respectively. The combination also showed a manageable toxicity profile and should be taken into consideration for the clinical management of patients with advanced PSCs and for further studies.

\section{Durvalumab Plus Tremelimumab in Head and Neck Squamous Cell Carcinoma (HNSCC)}

Head and neck squamous cell carcinoma (HNSCC) is among the ten most common cancers worldwide [49], with a 5-year OS rate of approximately $40-50 \%$ [50]. It is progressively increasing in incidence, and up to 60,000 new cases are diagnosed in the USA yearly [51]. Approximately $60 \%$ of the patients have locally advanced or metastatic disease at the time of diagnosis, with subsequent frequent locoregional or distant relapses [52]. The prognosis of patients with distant metastases is poor and the standard of care is palliative chemotherapy with combinations of cetuximab, platinum, and fluorouracil, with a median OS of 10 months and an ORR of $30 \%$ [53]. In addition to chemotherapy, local surgical debulking or radiation therapy may be used to achieve a prolonged PFS and to cure in some cases [54]. Immunotherapy is an attractive option because HNSCC is highly immunogenic, with up to $60 \%$ PDL-1 expression and high levels of intra-tumor regulatory T cell infiltrates $[55,56]$. Two studies were recently performed to evaluate the safety and efficacy of durvalumab and tremelimumab combined in comparison with monotherapy (CONDOR [22]) and/or SoC therapy (EAGLE [23]) in advanced HNSCC unsuccessfully treated with platinum-based chemotherapy (Table 3).

The CONDOR trial was a phase II study of 267 patients with recurrent and/or metastatic HNSCC, recruited over approximately 1 year from North America, Europe, and Asia Pacific centers [22]. In this study, the authors aimed to evaluate the safety and ORR of durvalumab and tremelimumab alone or in combination, while a phase III extension study is ongoing to evaluate the combination of these drugs as a second-line treatment in patients with PDL-1-high (TC $\geq 25 \%$ ) and PDL-1-low/negative (TC $<25 \%$ ) HNSCC. Grade 3/4 treatment-related adverse events occurred in 21 patients $(15.8 \%)$ treated with durvalumab + tremelimumab, 8 (12.3\%) treated with durvalumab, and $11(16.9 \%)$ treated with tremelimumab. ORR was $7.8 \%$ (95\% CI $3.78-13.79 \%)$ in the combination arm $(n=129), \quad 9.2 \% \quad(95 \%$ CI $3.46-19.02 \%)$ for 
Table 3 Oncological outcomes of the combination of durvalumab plus tremelimumab in comparison with other treatments in patients affected by head and neck cancers

\begin{tabular}{llllll}
\hline Author [References] & Value (95\% CI) & \multicolumn{2}{l}{ Treatments } & \multicolumn{2}{l}{ Comment } \\
\hline \multirow{2}{*}{ Siu et al. [22] } & & D $+\mathrm{T}$ & $\mathrm{D}$ & $\mathrm{T}$ & \\
& OS m & 7.6 & 6 & 5,5 & CONDOR trial \\
& PFS m & 2 & 1.9 & 1.9 & Treatment after one platinum-based cycle \\
& ORR \% & $7.8 \%$ & $9.20 \%$ & $1.60 \%$ & \\
& AE & $15.80 \%$ & $12.30 \%$ & $16.90 \%$ & \\
Ferris et al. [23] & $\mathrm{D}$ & $\mathrm{D}+\mathrm{T}$ & SoC & \\
& OS m & 7.6 & 6.5 & 8.3 & EAGLE trial \\
& PFS m & 2.1 & 2 & 3.7 & Treatment after one platinum-based cycle \\
& ORR \% & $17.90 \%$ & $18.20 \%$ & $17.30 \%$ & SoC = paclitaxel + cetuximab \\
\hline
\end{tabular}

$A E$ treatment-related adverse events, $C I$ confidence interval, $D$ durvalumab, ORR objective response rate, $O S m$ median overall survival, $P F S m$ median progression-free survival, $S o C$ standard of care, $T$ tremelimumab

durvalumab monotherapy $(n=65)$, and $1.6 \%$ $(95 \%$ CI $0.04-8.53 \%)$ for tremelimumab monotherapy $(n=63)$; median OS for all patients treated was 7.6 (95\% CI 4.9-10.6), 6.0 (95\% CI 4.0-11.3), and 5.5 (95\% CI 3.9-7.0) months, respectively. Therefore, durvalumab alone or with tremelimumab showed similar efficacy and a good manageability of the adverse effects.

The EAGLE study, conducted at 156 centers worldwide over approximately 1.5 years, recruited 736 patients who were randomly assigned to durvalumab monotherapy $(n=240)$, durvalumab plus tremelimumab combination therapy $(n=247)$, or conventional chemotherapy (paclitaxel plus cetuximab, $n=249$ ) [23]. The study did not demonstrate a statistically significant survival benefit for immunotherapy over single-agent SoC as second-line treatment. Indeed, no statistically significant improvements in OS were observed for durvalumab versus SoC (95\% CI 0.72-1.08) or durvalumab plus tremelimumab versus SoC (95\% CI 0.85-1.26). Interestingly, in this study a higher proportion of patients died within the first 3 months after randomization. However, patients who live beyond the initial treatment period appear to be the most likely to survive longer, given the higher survival rates for durvalumab monotherapy. Globally, the results of the CONDOR and EAGLE trials suggest that the combination of durvalumab plus tremelimumab does not introduce any consistent oncological advantage either in comparison to monotherapy with durvalumab nor in comparison with traditional chemotherapy, in patients with HNSCC.

\section{Durvalumab Plus Tremelimumab in Gastrointestinal Tumors}

Colorectal cancer (CRC) is the most common cancer of the gastrointestinal tract, with approximately two million new cases estimated in 2020 worldwide [57]. CRC is a well-studied malignancy for which extensive and heterogeneous genomic aberrations, well-defined risk factors, slow progression, and identifiable and treatable preneoplastic lesions have been described [58, 59]. Recent data have also validated the significant role of epigenetics in regulating the function of CRC cells [60-62].

Despite extensive knowledge of CRC biology and improvements in therapy and in population-based screening, it remains one of the hard-to-treat cancers considering the high 
frequency of metastases and recurrences after surgery, and frequent resistance to first- or second-line of treatment [63]. In fact, chemotherapy has been the main treatment in the past decades; survival rates have begun to increase with the introduction of targeted monoclonal antibodies [64]. Immunotherapy has shown promising results in several gastrointestinal cancers, including gastroesophageal cancer, hepatocellular carcinoma, and CRC. Regarding CRC, pemrolizumab and ipilimumab are currently used in clinical practice for the treatment of specific subsets of patients with CRC, who have progressed through first-line chemotherapy [65]. Numerous ongoing clinical trials are investigating further agents and their combinations.

Recently, Segal et al. performed a singlecenter phase II study including 24 patients with chemotherapy-refractory with mismatch repair (MMR)-proficient metastatic CRC treated with durvalumab plus tremelimumab plus radiotherapy (Table 4) [24]. In this study, the ORR was $8.3 \%$ (95\% CI 1.0-27.0), the median PFS was 1.8 (95\% CI 1.7-1.9) months, and the median OS was $11.4 \quad(95 \%$ CI 10.1-17.4) months. Twenty-five percent of patients had grade 3/4 treatment-related adverse events. The scheme did not meet the prespecified endpoint criteria, even if some cases of systemic immune enhancement and regression were observed in non-irradiated lesions. The study showed that the combination durvalumab and tremelimumab in addition to radiotherapy is feasible, but its clinical advantages need to be further evaluated in future studies.

Kelly et al. tested durvalumab and tremelimumab in patients with gastric and gastroesophageal junction (GEJ) cancer. These cancers are among the eight most common global cancers worldwide [66], and their incidence has increased significantly in Western countries in the last decades [67]. In addition, approximately $40 \%$ of patients with gastric cancer/GEJ cancer express PDL-1 on tumor and immune cells $[68,69]$. Kelly et al. performed a randomized, multicenter, open-label, phase Ib/II trial to study the clinical benefits of durvalumab and tremelimumab in combination and as monotherapy in chemotherapy-refractory advanced gastric cancer/GEJ carcinoma [25], in particular patients who progressed after two or more lines of systemic platinum- or fluoropyrimidine-based chemotherapy, and presence of a measurable biopsy-eligible lesion. ORR and PFS at 6 and 12 months were evaluated, and no significant differences were observed between treatment arms. Nevertheless, the combination of durvalumab and tremelimumab demonstrated numerically higher ORR than durvalumab monotherapy. The authors highlighted that although response rates with durvalumab or tremelimumab monotherapy or in combination were low, the combination approach resulted in a 12-month OS rate of approximately $37 \%$ (95\% CI 19.6-54.6\%).

Our search identified two studies performed in patients with pancreatic cancer [26, 27]. Pancreatic ductal adenocarcinoma (PDAC) is the third leading cause of cancer-related death in the USA, having recently surpassed breast cancer [70], and is projected to become the second leading cause of cancer death by 2030 [71]. It has an extremely poor prognosis, with a 5 -year survival rate at approximately $7 \%$ $[72,73]$. The only curative treatment is complete surgical resection, but unfortunately only a small proportion of patients have surgically treatable disease. Although FOLFIRINOX or gemcitabine-based chemotherapies have improved the OS, they only marginally improve survival [74-76]. A distinctive feature of PDAC is its immunosuppressive microenvironment making it an attractive candidate for immunotherapy, and some anti-PD-1 agents have been studied or are currently under investigation.

The first study that investigated durvalumab plus tremelimumab in patients with previously treated metastatic disease was published by O'Reilly et al. in 2019 [26]. The study was carried out between November 2015 and March 2017 and included 65 patients who had previously received only first-line treatment with fluorouracil or gemcitabine at 21 centers in six countries. The ORR was $3.1 \% \quad(95 \%$ CI 0.08-16.22) for patients receiving combination therapy and $0 \% \quad(95 \%$ CI $0.00-10.58)$ for patients receiving monotherapy. The median PFS was 1.5 (95\% CI 1.2-1.5) months in both 
Table 4 Oncological outcomes of the combination of durvalumab plus tremelimumab in comparison with other treatments in patients affected by gastrointestinal cancers

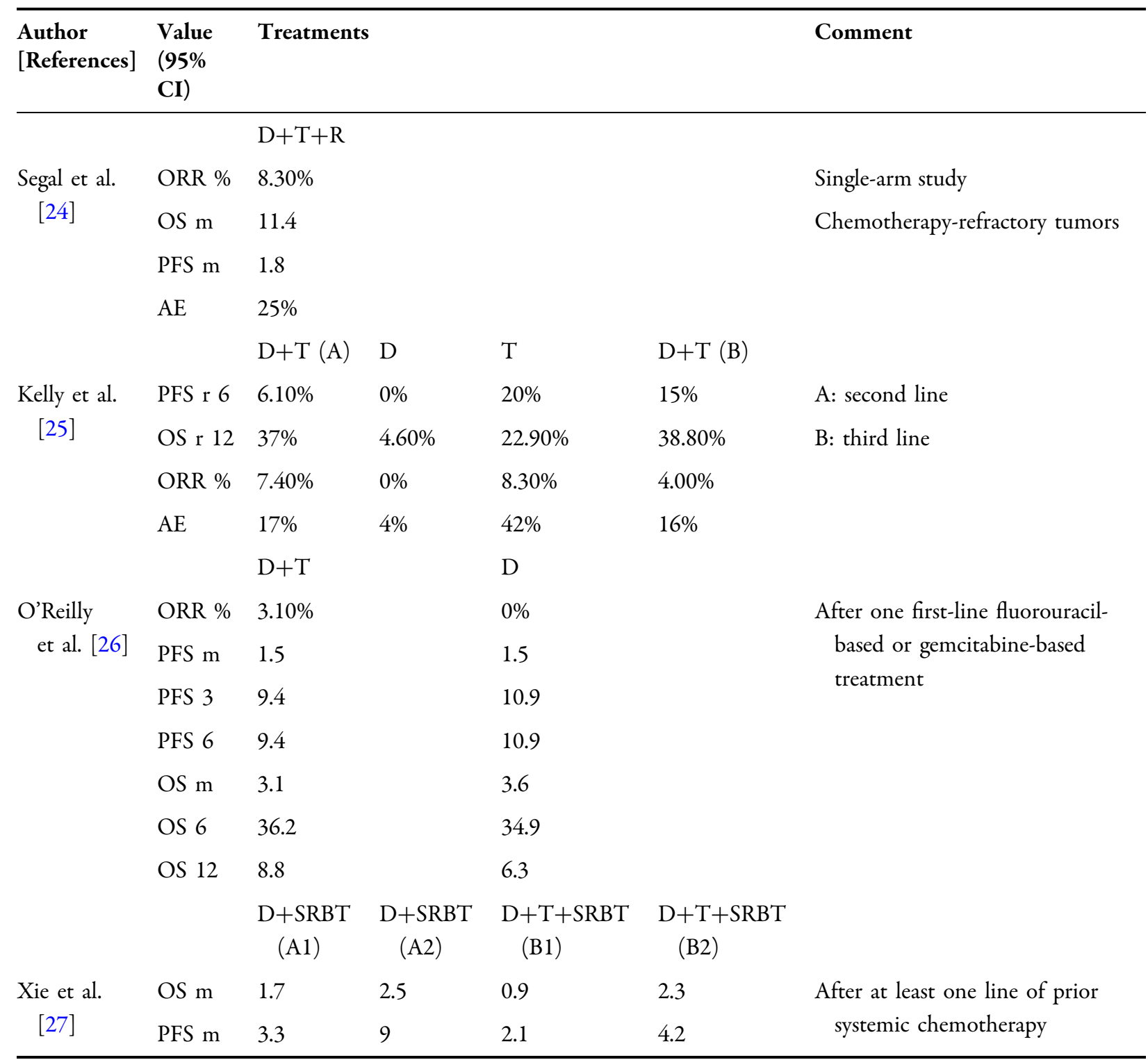

$A 1 / A 2$ radiation dose levels 8 Gy in 1 fraction on day, $A E$ treatment-related adverse events, $B 1 / B 2$ radiation dose levels $25 \mathrm{~Gy}$ in 5 fractions over 5 days, $D$ durvalumab, ORR\% objective response rate, $O S 6 / 12$ overall survival for $6 / 12$ months, OS $m$ median overall survival, $O S$ r 12 overall survival rate for 12 months, $P F S 3 / 6$ progression-free survival for $3 / 6$ months, $P F S m$ median progression-free survival, PFS $r 6$ progression-free survival rate for 6 months, $R$ radiotherapy, SBRT stereotactic body radiation therapy, $T$ tremelimumab

treatments, and the median OS was 3.1 (95\% CI 2.2-6.1) months in combination therapy compared with 3.6 (95\% CI 2.7-6.1) months in monotherapy. Despite a good tolerability of the treatments, the study was stopped because the initial results did not meet the prespecified endpoint (10\% ORR in both arms).

The second study published in 2020 by Xie et al. and aimed to evaluate the safety of the combination durvalumab plus tremelimumab 
or durvalumab alone, both associated with SBRT in patients with metastatic PDAC [27]. The rationale is based on evidences that radiation therapy, which is used in the management of advanced stages of pancreatic cancer, increases the peripheral antitumor immunity [77-79]. Xie et al. enrolled 59 patients, including 39 evaluable patients who had received at least one line of prior systemic chemotherapy. Two groups received durvalumab plus a different fraction of SBRT (A1 and A2), while two further groups received durvalumab plus tremelimumab and the same fractions of SBRT (B1 and B2). The primary objective of the study was to evaluate the safety and feasibility of the treatments, and it was continued until unacceptable toxicity or disease progression occurred. The ORR was 5.1\%, and better PFS and OS were observed in the B2 group of patients (durvalumab plus tremelimumab plus $25 \mathrm{~Gy}$ in five fractions over 5 days), with respectively 2.3 (95\% CI 1.9-3.4) and 4.2 (95\% CI 2.9-9.3) months. The authors concluded that the combination of ICIs and SBRT had an acceptable safety profile, and a good clinical benefit in patients with metastatic PDAC. This evidence resizes the initial disappointment that followed the study by O'Reilly et al., and opens a new horizon for research regarding the use of durvalumab plus tremelimumab in patients with PDAC.

Finally, we identified one study reporting on the use of the combination in biliary tract cancers (BTC), which comprise intrahepatic cholangiocarcinoma (ICC), extrahepatic ICC, and gallbladder carcinoma, all with distinct biological, molecular, and oncological features [80]. These malignancies are again characterized by extremely high mortality rates, and only a few therapeutic options are currently available for patients affected by advanced stage disease. Active research is currently ongoing to identify novel targeted therapies and immunotherapeutic agents in this setting [81-86] The IMMUNOBIL PRODIGE 57 trial was designed to evaluate the efficacy and safety of durvalumab plus tremelimumab, with or without paclitaxel, in patients with advanced BTC after failure of platinum-based chemotherapy [28]. Twenty patients in total, 10 in each arm, were enrolled.
No dose-limiting toxicities (DLTs) were observed in the arm without paclitaxel, while six DLTs were observed in that including all three medications, leading to study discontinuation. Therefore, the ongoing phase II study will evaluate only the combination of durvalumab and tremelimumab.

\section{Durvalumab Plus Tremelimumab in Urogenital Tumors}

Bladder cancer represents a major cause of morbidity and mortality worldwide, with an estimated 180,500 new cases and 38,200 deaths in Europe each year [87]. Urothelial carcinoma (UC) accounts for approximately 90\% of all bladder cancers [88]. Gao et al. [29] carried out a pilot study investigating the neoadjuvant combination of durvalumab plus tremelimumab in 28 cisplatin-unsuitable patients with high-risk tumors (Table 5). The primary endpoint was safety and was observed in $21 \%$ of patients. They also reported a pathologic complete response in $37.5 \%$ of the cases, $88.8 \%$ (standard error $\mathrm{SE}=6.1 \%)$ 1-year $\mathrm{OS}$, and $82.8 \%(\mathrm{SE}=$ $7.9 \%)$ recurrence-free survival at 1 year in the 24 patients who underwent cystectomy. The authors advocate that the issue warrants further investigation.

Further evidence came from the DANUBE trial, an open-label, randomized, controlled, phase III study conducted at 224 centers in 23 countries, where the authors evaluated the OS of patients who received durvalumab, with or without tremelimumab, as first-line treatment for metastatic urothelial carcinoma [30]. The 1032 patients enrolled randomly to receive durvalumab as monotherapy $(n=346)$, durvalumab plus tremelimumab $(n=342)$, or SoC chemotherapy $(n=344$; gemcitabine plus cisplatin or gemcitabine plus carboplatin). In the population with elevated PDL-1, the median OS was 14.4 (95\% CI 10.4-17.3) months in the durvalumab monotherapy group, compared with 12.1 (95\% CI 10.4-15.0) months in the chemotherapy group. The median OS in the durvalumab plus tremelimumab group was 15.1 (95\% CI 13.1-18.0) months. Nevertheless, serious treatment-related adverse events occurred 
Table 5 Oncological outcomes of the combination of durvalumab plus tremelimumab in comparison with other treatments in patients affected by urogenital cancers

\begin{tabular}{|c|c|c|c|c|}
\hline $\begin{array}{l}\text { Author } \\
\text { [References] }\end{array}$ & $\begin{array}{l}\text { Value }(95 \% \\
\text { CI) }\end{array}$ & Treatments & & Comment \\
\hline & & $\mathrm{D}+\mathrm{T}$ & & \\
\hline \multirow[t]{4}{*}{ Gao et al. [29] } & PCR & $37.50 \%$ & & Neoadjuvant \\
\hline & OS $1 y$ & $88.80 \%$ & & \\
\hline & RFS 1y & $82.80 \%$ & & \\
\hline & & $\mathrm{D} \quad \mathrm{D}+\mathrm{T}$ & SoC & \\
\hline \multirow[t]{4}{*}{ Powles et al. [30] } & OS m & $14.4 \quad 15.1$ & 12.1 & DANUBE trial \\
\hline & $\mathrm{AE}$ & $9 \% \quad 23 \%$ & $16 \%$ & First line \\
\hline & & & & $\begin{array}{l}\text { SoC: gemcitabine plus cisplatin or gemcitabine plus } \\
\text { carboplatin }\end{array}$ \\
\hline & & $\mathrm{D}+\mathrm{T}$ & & \\
\hline \multirow[t]{4}{*}{ Sarfaty et al. [31] } & OS $r$ & $6.97 \%$ & & Single-arm study \\
\hline & PFS $r$ & $1.80 \%$ & & Treated and untreated patients \\
\hline & $\mathrm{AE}$ & $38.40 \%$ & & \\
\hline & & $\mathrm{D}+\mathrm{T}$ & $\mathrm{D}$ & \\
\hline \multirow[t]{4}{*}{ Necchi et al. [32] } & PFS 3 & 18.2 & 0 & APACHE trial \\
\hline & PFS m & 1.7 & 1.5 & \\
\hline & OS 6 & 41.6 & 29.5 & \\
\hline & OS m & 3.1 & 3.1 & \\
\hline
\end{tabular}

$A E$ treatment-related adverse events, $D$ durvalumab, $O S 6 / 1 Y$ overall survival for 6 months or 1 year, $O S m$ median overall survival, $O S r$ overall survival rate, $P C R$ pathologic complete response, $P F S 3$ progression-free survival for 3 months, PFS $m$ median progression-free survival, $P F S r$ progression-free survival rate, $R F S$ recurrence-free survival, $S o C$ standard of care, $T$ tremelimumab

in $9 \%$ in the durvalumab group, $23 \%$ in the durvalumab plus tremelimumab group, and $16 \%$ in the chemotherapy group. The study did not meet any of the primary endpoints, suggesting that the issue for future research is a better pathological or molecular classification of the patients with metastatic urothelial carcinoma who might benefit from ICIs.

Sarfaty et al. performed a study on patients with non-urothelial carcinoma, the smaller counterpart of urologic cancers that includes several histotypes, like squamous cell carcinoma (SCC), adenocarcinoma (ADC), and small cell/ neuroendocrine carcinoma (NE) [89]. In this open-label phase II study, 13 patients (seven NE, three SCC, and three ADC) were treated with durvalumab and tremelimumab [31]. No significant responses were observed; the median PFS was 1.8 (95\% CI 1.25-not reached) months, and the median OS was 6.97 (95\% CI 4.34-not reached) months. Grade 3/4 treatment-related adverse events occurred in $38.4 \%$ of patients. The authors concluded that in patients with non-urothelial urinary cancer, durvalumab combined with tremelimumab lacked clinical 
activity, even if they demonstrated a manageable safety profile.

Similar conclusions were drawn also by Necchi et al. who performed a study on patients with germ cell tumors (GCTs), which were shown able to express PDL-1 in past studies [90, 91]. Necchi et al. [32] with the APACHE trial, an open-label randomized phase II study on 22 patients who underwent treatment with durvalumab alone or durvalumab plus tremelimumab, showed that in durvalumab monotherapy a significant number of patients showed strong disease progression, while combination immunotherapy is a therapeutic option that could be used in selected cases, although predictive biomarkers are lacking.

Globally, it appears clear that in urogenital tumors the combination of durvalumab plus tremelimumab showed relevant limitations and it seems that it could be used in specific subsets of patients with particular biological and molecular features, which remain to be elucidated.

\section{CONCLUSIONS AND FUTURE PERSPECTIVES}

The results of the studies included in this review globally suggest that the combination of durvalumab with tremelimumab does not seem to bring consistent advantages, even in subsets of patients with demonstrated responsiveness in other immunotherapies, like NSCLC and HNSCC. Certainly, it should be kept in mind that some of the studies discussed have relevant methodological limitations, like the single-arm, non-randomized design, and the small number of patients enrolled. Nevertheless, durvalumab plus tremelimumab showed poor results also in some of the well-designed studies retrieved. Further studies investigating this combination of drugs are ongoing, and the results are awaited to draw better conclusions.

The KGOG 3046 [92] is a phase II single-arm study initiated in Korea to evaluate the impact of this treatment on patients with advanced ovarian cancer. Bahig et al. published recently the protocol of another ongoing study designed to assess the safety and efficacy of a triple treatment combination consisting of the administration of durvalumab and tremelimumab in combination with SBRT in metastatic HNSCC [93]. Kelley et al. presented recently the results of a phase I/II study, in which the combination of durvalumab with tremelimumab was evaluated in a total of 40 patients with advanced hepatocellular carcinoma (HCC) in comparison with the respective monotherapies [94]. Preliminary results, again, were not particularly encouraging, especially in terms of toxicity; further data are expected to better understand the role of these medications in HCC. Other trials are currently ongoing in pediatric (NCT03837899), neuroendocrine (NCT03095274), thyroid (NCT03753919), prostate (NCT03204812), breast (NCT03132467), and other cancers. Data from real-life studies are also warranted for a better evaluation of the clinical usefulness of the combination durvalumab-tremelimumab in specific subsets of cancers and hopefully a better classification of the patients according to biomarkers which help to identify good responders and patients who gain real prognostic benefits.

\section{ACKNOWLEDGEMENTS}

Funding. Open access funding provided by Università degli Studi di Sassari within the CRUI-CARE Agreement. The Journal's Rapid Service fee was funded by the authors.

Authorship. All named authors meet the International Committee of Medical Journal Editors (ICMJE) criteria for authorship for this article, take responsibility for the integrity of the work as a whole, and have given their approval for this version to be published.

Authorship Contributions. Caterina Arru, Maria Rosaria De Miglio, and Panagiotis Paliogiannis: conceptualization, study design and supervision; Antonio Cossu, Maria Rosaria De Miglio, Ciriaco Carru, and Anglelo Zinellu: literature search, database curation, drafting and critical revision of parts of the manuscript; 
Maria Rosaria and Panagiotis Paliogiannis: revision of the final version of the manuscript.

Disclosures. All the named authors declare no conflict of interest.

Compliance with Ethics Guidelines. This article is based on previously conducted studies and does not contain any new studies with human participants or animals performed by any of the authors.

Data Availability. The data of the systematic literature search are available by the corresponding author upon reasonable request.

Open Access. This article is licensed under a Creative Commons Attribution-NonCommercial 4.0 International License, which permits any non-commercial use, sharing, adaptation, distribution and reproduction in any medium or format, as long as you give appropriate credit to the original author(s) and the source, provide a link to the Creative Commons licence, and indicate if changes were made. The images or other third party material in this article are included in the article's Creative Commons licence, unless indicated otherwise in a credit line to the material. If material is not included in the article's Creative Commons licence and your intended use is not permitted by statutory regulation or exceeds the permitted use, you will need to obtain permission directly from the copyright holder. To view a copy of this licence, visit http://creativecommons.org/licenses/by$\mathrm{nc} / 4.0 /$.

\section{REFERENCES}

1. Renrick AN, Dunbar ZT, Shanker A. Update on the current revolution in cancer immunotherapy. Immunotherapy. 2019;11:15-20.

2. Leach DR, Krummel MF, Allison JP. Enhancement of antitumor immunity by CTLA- 4 blockade. Science. 1996;271:1734-6.

3. Rohatgi A, Kirkwood JM. Beyond PD-1: the next frontier for immunotherapy in melanoma. Front Oncol. 2021;11:76.
4. Ribas A, Wolchok JD. Cancer immunotherapy using checkpoint blockade. Science. 2018;359:1350-5.

5. Olofsson Bagge R, Ny L, Ascierto PA, et al. The efficacy of immunotherapy for in-transit metastases of melanoma: an analysis of randomized controlled trials. Melanoma Res. 2021;31:181-5.

6. Putzu C, Cortinovis DL, Colonese F, et al. Blood cell count indexes as predictors of outcomes in advanced non-small-cell lung cancer patients treated with nivolumab. Cancer Immunol Immunother. 2018;67:1349-53.

7. Lee HT, Lee JY, Lim H, et al. Molecular mechanism of PD-1/PD-L1 blockade via anti-PD-L1 antibodies atezolizumab and durvalumab. Sci Rep. 2017;7: $1-12$.

8. Alvarez-Argote J, Dasanu CA. Durvalumab in cancer medicine: a comprehensive review. Expert Opin Biol Ther. 2019;19:927-35.

9. Murakami S. Durvalumab for the treatment of nonsmall cell lung cancer. Expert Rev Anticancer Ther. 2019;19:1009-16.

10. Blank CU, Enk A. Therapeutic use of anti-CTLA-4 antibodies. Int Immunol. 2015;27:3-10.

11. Calabrò L, Ceresoli GL, di Pietro A, et al. CTLA4 blockade in mesothelioma: finally a competing strategy over cytotoxic/target therapy? Cancer Immunol Immunother. 2015;64:105-12.

12. Chung KY, Gore I, Fong L, et al. Phase II study of the anti-cytotoxic T-lymphocyte-associated antigen 4 monoclonal antibody, tremelimumab, in patients with refractory metastatic colorectal cancer. J Clin Oncol. 2010;28:3485-90.

13. Comin-Anduix B, Escuin-Ordinas H, Ibarrondo FJ. Tremelimumab: research and clinical development. Onco Targets Ther. 2016;9:1767-76.

14. Antonia S, Goldberg SB, Balmanoukian A, et al. Safety and antitumour activity of durvalumab plus tremelimumab in non-small cell lung cancer: a multicentre, phase $1 \mathrm{~b}$ study. Lancet Oncol. 2016;17:299-308.

15. Rizvi NA, Cho BC, Reinmuth N, et al. Durvalumab with or without tremelimumab vs standard chemotherapy in first-line treatment of metastatic non-small cell lung cancer: the MYSTIC phase 3 randomized clinical trial. JAMA Oncol. 2020;6: 661-74.

16. Planchard D, Reinmuth N, Orlov S, et al. ARCTIC: durvalumab with or without tremelimumab as third-line or later treatment of metastatic nonsmall-cell lung cancer. Ann Oncol. 2020;31:609-18. 
17. Juergens RA, Hao D, Ellis PM, et al. A phase IB study of durvalumab with or without tremelimumab and platinum-doublet chemotherapy in advanced solid tumours: Canadian Cancer Trials Group Study IND226. Lung Cancer. 2020;143:1-11.

18. Goldman JW, Dvorkin M, Chen Y, et al. Durvalumab, with or without tremelimumab, plus platinum-etoposide versus platinum-etoposide alone in first-line treatment of extensive-stage small-cell lung cancer (CASPIAN): updated results from a randomised, controlled, open-label, phase 3 trial. Lancet Oncol. 2021;22:51-65.

19. Pakkala S, Higgins K, Chen Z, et al. Durvalumab and tremelimumab with or without stereotactic body radiation therapy in relapsed small cell lung cancer: a randomized phase II study. J Immunother Cancer. 2020;8:e001302.

20. Calabrò L, Morra A, Giannarelli D, et al. Tremelimumab combined with durvalumab in patients with mesothelioma (NIBIT-MESO-1): an open-label, non-randomised, phase 2 study. Lancet Respir Med. 2018;6:451-60.

21. Kim M, Keam B, Ock CY, et al. Phase II study of durvalumab and tremelimumab in pulmonary sarcomatoid carcinoma: KCSG-LU16-07. Thorac Cancer. 2020;11:3482-9.

22. Siu LL, Even C, Mesía R, et al. Safety and efficacy of durvalumab with or without tremelimumab in patients with PD-L1-low/negative recurrent or metastatic HNSCC: the phase 2 CONDOR randomized clinical trial. JAMA Oncol. 2019;5: 195-203.

23. Ferris RL, Haddad R, Even C, et al. Durvalumab with or without tremelimumab in patients with recurrent or metastatic head and neck squamous cell carcinoma: EAGLE, a randomized, open-label phase III study. Ann Oncol. 2020;31:942-50.

24. Segal NH, Cercek A, Ku GY, et al. Phase II single arm study of durvalumab and tremelimumab with concurrent radiotherapy in patients with mismatch repair proficient metastatic colorectal cancer. Clin Cancer Res. 2021;27:2200-8.

25. Kelly RJ, Lee J, Bang YJ, et al. Safety and efficacy of durvalumab and tremelimumab alone or in combination in patients with advanced gastric and gastroesophageal junction adenocarcinoma. Clin Cancer Res. 2020;26:846-54.

26. O'Reilly EM, Oh DY, Dhani N, et al. Durvalumab with or without tremelimumab for patients with metastatic pancreatic ductal adenocarcinoma: a phase 2 randomized clinical trial. JAMA Oncol. 2019;5:1431-8.
27. Xie C, Duffy AG, Brar G, et al. Immune checkpoint blockade in combination with stereotactic body radiotherapy in patients with metastatic pancreatic ductal adenocarcinoma. Clin Cancer Res. 2020;26: 2318-26.

28. Boilève A, Hilmi M, Gougis $\mathrm{P}$, et al. Triplet combination of durvalumab, tremelimumab, and paclitaxel in biliary tract carcinomas: safety run-in results of the randomized IMMUNOBIL PRODIGE 57 phase II trial. Eur J Cancer. 2021;143:55-63.

29. Gao J, Navai N, Alhalabi O, et al. Neoadjuvant PDL1 plus CTLA-4 blockade in patients with cisplatinineligible operable high-risk urothelial carcinoma. Nat Med. 2020;26:1845-51.

30. Powles T, van der Heijden MS, Castellano D, et al. Durvalumab alone and durvalumab plus tremelimumab versus chemotherapy in previously untreated patients with unresectable, locally advanced or metastatic urothelial carcinoma (DANUBE): a randomised, open-label, multicentre, phase 3 trial. Lancet Oncol. 2020;21:1574-88.

31. Sarfaty M, Whiting K, Teo MY, et al. A phase II trial of durvalumab and tremelimumab in metastatic, non-urothelial carcinoma of the urinary tract. Cancer Med. 2020;10:1074.

32. Necchi A, Giannatempo P, Raggi D, et al. An openlabel randomized phase 2 study of durvalumab alone or in combination with tremelimumab in patients with advanced germ cell tumors (APACHE): results from the first planned interim analysis. Eur Urol. 2019;75:201-3.

33. Global Cancer Oservatory. Cancer Fact Sheets. Lung. Avaialble at: https:/gco.iarc.fr/today/data/ factsheets/cancers/15-Lung-fact-sheet.pdf. Accessed 05 Mar 2021.

34. Paliogiannis P, Attene F, Cossu A, et al. Lung cancer epidemiology in North Sardinia, Italy. Multidiscip Respir Med. 2013. https://doi.org/10.4081/mrm. 2013.543.

35. Remon J, Aldea M, Besse B, et al. Small cell lung cancer: a slightly less orphan disease after immunotherapy. Ann Oncol. 2021. https://doi.org/ 10.1016/j.annonc.2021.02.025.

36. Fois SS, Paliogiannis P, Zinellu A, et al. Molecular epidemiology of the main druggable genetic alterations in non-small cell lung cancer. Int J Mol Sci. 2021;22:1-19.

37. Antonia SJ, Villegas A, Daniel D, et al. Overall survival with durvalumab after chemoradiotherapy in stage III NSCLC. N Engl J Med. 2018;379:2342-50. 
38. Antonia SJ, Villegas A, Daniel D, et al. Durvalumab after chemoradiotherapy in stage III non-small-cell lung cancer. N Engl J Med. 2017;377:1919-29.

39. Chaft J, Cho BC, Ahn M-J, et al. Abstract CT113: safety and activity of second-line durvalumab + tremelimumab in non-squamous advanced NSCLC. In: Cancer research. Washington: American Association for Cancer Research (AACR); 2018. p. CT113.

40. Byers LA, Rudin CM. Small cell lung cancer: Where do we go from here? Cancer. 2015;121:664-72.

41. Delgermaa V, Takahashi K, Park EK, et al. Les décés mondiaux par mésothéliome rapportés á l'Organisation mondiale de la Santé entre 1994 et 2008. Bull World Health Organ. 1994;2011:89.

42. Bibby AC, Tsim S, Kanellakis N, et al. Malignant pleural mesothelioma: an update on investigation, diagnosis and treatment. Eur Respir Rev. 2016;25: 472-86.

43. Pala C, Paliogiannis P, Serventi F, et al. Multimodality approach to malignant pleural mesothelioma. A case report. Ann Ital Chir. 2010;81:37-40.

44. Brambilla E, Travis WD, Colby TV, et al. The new World Health Organization classification of lung tumours. Eur Respir J. 2001;18:1059-68.

45. Travis WD. Sarcomatoid neoplasms of the lung and pleura. Arch Pathol Lab Med. 2010;134:1645-58.

46. Bae HM, Min HS, Lee $\mathrm{SH}$, et al. Palliative chemotherapy for pulmonary pleomorphic carcinoma. Lung Cancer. 2007;58:112-5.

47. Lee J, Jung HA, Kim Y, et al. Efficacy of mesna, doxorubicin, ifosfamide, and dacarbazine (MAID) in patients with advanced pulmonary pleomorphic carcinoma. Lung Cancer. 2018;122:160-4.

48. Yendamuri S, Caty L, Pine M, et al. Outcomes of sarcomatoid carcinoma of the lung: a Surveillance, Epidemiology, and End Results database analysis. Surgery. 2012;152:397-402.

49. Fitzmaurice C, Abate D, Abbasi N, et al. Global, regional, and national cancer incidence, mortality, years of life lost, years lived with disability, and disability-adjusted life-years for 29 cancer groups, 1990 to 2017. JAMA Oncol. 2019;5:1749.

50. Mandal R, Şenbabaoğlu Y, Desrichard A, et al. The head and neck cancer immune landscape and its immunotherapeutic implications. JCI Insight. 2016. https://doi.org/10.1172/jci.insight.89829.

51. Siegel RL, Miller KD, Jemal A. Cancer statistics, 2016. CA Cancer J Clin. 2016;66:7-30.
52. Vermorken JB, Specenier P. Optimal treatment for recurrent/metastatic head and neck cancer. Ann Oncol. 2010. https://doi.org/10.1093/annonc/ mdq453.

53. Vermorken JB, Mesia R, Rivera F, et al. Platinumbased chemotherapy plus cetuximab in head and neck cancer. N Engl J Med. 2008;359:1116-27.

54. Weichselbaum RR, Hellman S. Oligometastases revisited. Nat Rev Clin Oncol. 2011;8:378-82.

55. Concha-Benavente F, Srivastava RM, Trivedi S, et al. Identification of the cell-intrinsic and -extrinsic pathways downstream of EGFR and IFN $\gamma$ that induce PD-L1 expression in head and neck Cancer. Cancer Res. 2016;76:1031-43.

56. Badoual C, Hans S, Merillon N, et al. PD-1-expressing tumor-infiltrating $\mathrm{T}$ cells are a favorable prognostic biomarker in HPV-associated head and neck cancer. Cancer Res. 2013;73:128-38.

57. Global Cancer Oservatory. Cancer Fact Sheets. Colorectum and Anus. Avaialble at: https://gco.iarc. $\mathrm{fr} /$ today/data/factsheets/cancers/10_8_9-

Colorectum-fact-sheet.pdf. Accessed 05 Mar 2021.

58. Pira G, Uva P, Scanu AM, et al. Landscape of transcriptome variations uncovering known and novel driver events in colorectal carcinoma. Sci Rep. 2020;10:432.

59. Uleri E, Piu C, Caocci M, et al. Multiple signatures of the JC polyomavirus in paired normal and altered colorectal mucosa indicate a link with human colorectal cancer, but not with cancer progression. Int J Mol Sci. 2019;20:5965.

60. Angius A, Pira G, Scanu AM, et al. Microrna-425-5p expression affects BRAF/RAS/MAPK pathways in colorectal cancers. Int J Med Sci. 2019;16:1480-91.

61. Angius A, Uva P, Pira G, et al. Integrated analysis of miRNA and mRNA endorses a twenty miRNAs signature for colorectal carcinoma. Int $\mathrm{J}$ Mol Sci. 2019;20:4067.

62. Fadda A, Gentilini D, Moi L, et al. Colorectal cancer early methylation alterations affect the crosstalk between cell and surrounding environment, tracing a biomarker signature specific for this tumor. Int $\mathrm{J}$ Cancer. 2018;143:907-20.

63. Palmieri G, Paliogiannis P, Scognamillo F, et al. Colorectal cancer epidemiology in an area with a spontaneous screening program. Acta Med Mediter. 2013;29:231.

64. Paliogiannis P, Cossu A, Tanda F, et al. KRAS mutational concordance between primary and 
metastatic colorectal adenocarcinoma. Oncol Lett. 2014;8:1422-6.

65. Golshani G, Zhang Y. Advances in immunotherapy for colorectal cancer: a review. Therap Adv Gastroenterol. 2020. https://doi.org/10.1177/ 1756284820917527.

66. Torre LA, Siegel RL, Ward EM, Jemal A. Global cancer incidence and mortality rates and trends - an update. Cancer Epidemiol Biomarkers Prev. 2016;25:16-27.

67. Buas MF, Vaughan TL. Epidemiology and risk factors for gastroesophageal junction tumors: Understanding the rising incidence of this disease. Semin Radiat Oncol. 2013;23:3-9.

68. Fuchs CS, Doi T, Jang RW, et al. Safety and efficacy of pembrolizumab monotherapy in patients with previously treated advanced gastric and gastroesophageal junction cancer: phase 2 clinical KEYNOTE-059 trial. JAMA Oncol. 2018;4:e180013.

69. Thompson ED, Zahurak M, Murphy A, et al. Patterns of PD-L1 expression and CD8 T cell infiltration in gastric adenocarcinomas and associated immune stroma. Gut. 2016;66:794-801.

70. Siegel RL, Miller KD, Jemal A. Cancer statistics, 2017. CA Cancer J Clin. 2017;67:7-30.

71. Rahib L, Smith BD, Aizenberg R, et al. Projecting cancer incidence and deaths to 2030: the unexpected burden of thyroid, liver, and pancreas cancers in the United States. Cancer Res. 2014;74: 2913-21.

72. Ilic M, Ilic I. Epidemiology of pancreatic cancer. World J Gastroenterol. 2016;22:9694-705.

73. Paliogiannis P. Pancreatic cancer burden in North Sardinia, Italy. Acta Medica Mediterr. 2015;31:413.

74. Conroy T, Hammel P, Hebbar M, et al. FOLFIRINOX or gemcitabine as adjuvant therapy for pancreatic cancer. N Engl J Med. 2018;379:2395-406.

75. Dhir M, Zenati MS, Hamad A, et al. FOLFIRINOX versus gemcitabine/Nab-paclitaxel for neoadjuvant treatment of resectable and borderline resectable pancreatic head adenocarcinoma. Ann Surg Oncol. 2018;25:1896-903.

76. Von Hoff DD, Ervin T, Arena FP, et al. Increased survival in pancreatic cancer with nab-paclitaxel plus gemcitabine. N Engl J Med. 2013;369: 1691-703.

77. Blanquicett C, Saif MW, Buchsbaum DJ, et al. Antitumor efficacy of capecitabine and celecoxib in irradiated and lead-shielded, contralateral human
BxPC-3 pancreatic cancer xenografts: clinical implications of abscopal effects. Clin Cancer Res. 2005;11:8773-81.

78. Demaria $\mathrm{S}, \mathrm{Ng} \mathrm{B}$, Devitt $\mathrm{ML}$, et al. Ionizing radiation inhibition of distant untreated tumors (abscopal effect) is immune mediated. Int J Radiat Oncol Biol Phys. 2004;58:862-70.

79. Lee Y, Auh SL, Wang Y, et al. Therapeutic effects of ablative radiation on local tumor require CD8+ T cells: changing strategies for cancer treatment. Blood. 2009;114:589-95.

80. Valle JW, Borbath I, Khan SA, et al. Biliary cancer: ESMO clinical practice guidelines for diagnosis, treatment and follow-up. Ann Oncol. 2016;27: v28-37.

81. Simile MM, Bagella P, Vidili G, et al. Targeted therapies in cholangiocarcinoma: emerging evidence from clinical trials. Medicina. 2019;55:42.

82. Gou M, Zhang Y, Si H, Dai G. Efficacy and safety of nivolumab for metastatic biliary tract cancer. Onco Targets Ther. 2019;12:861-7.

83. Bang Y-J, Ueno M, Malka D, et al. Pembrolizumab (pembro) for advanced biliary adenocarcinoma: results from the KEYNOTE-028 (KNO28) and KEYNOTE-158 (KN158) basket studies. J Clin Oncol. 2019;37:4079-4079.

84. Bang YJ, Doi T, De BF, et al. 525 Safety and efficacy of pembrolizumab (MK-3475) in patients (pts) with advanced biliary tract cancer: interim results of KEYNOTE-028. Eur J Cancer. 2015;51:S112.

85. Kim RD, Kim DW, Alese OB, et al. A phase II study of nivolumab in patients with advanced refractory biliary tract cancers (BTC). J Clin Oncol. 2019;37: 4097-4097.

86. Alshari OM, Dawaymeh TA, Tashtush NA, et al. Completely resolved advanced biliary tract cancer after treatment by pembrolizumab: a report of two cases. Onco Targets Ther Vol. 2019;12:5293-8.

87. Burger M, Catto JWF, Dalbagni G, et al. Epidemiology and risk factors of urothelial bladder cancer. Eur Urol. 2013;63:234-41.

88. Wong MCS, Fung FDH, Leung C, et al. The global epidemiology of bladder cancer: a joinpoint regression analysis of its incidence and mortality trends and projection. Sci Rep. 2018;8:1129.

89. Al-Ahmadie H, Iyer G. Molecular alterations in the pathogenesis of bladder cancer subtypes and urothelial carcinoma variants. Cham: Springer; 2018. p. 65-83. 
90. Fankhauser CD, Curioni-Fontecedro A, Allmann V, et al. Frequent PD-L1 expression in testicular germ cell tumors. Br J Cancer. 2015;113:411-3.

91. Cierna Z, Mego M, Miskovska V, et al. Prognostic value of programmed-death-1 receptor (PD-1) and its ligand 1 (PD-L1) in testicular germ cell tumors. Ann Oncol. 2016;27:300-5.

92. Lee JY, Kim JW, Lim MC, et al. A phase II study of neoadjuvant chemotherapy plus durvalumab and tremelimumab in advanced-stage ovarian cancer: a Korean gynecologic oncology group study (KGOG
3046), TRUD-D. J Gynecol Oncol. 2019. https://doi. org/10.3802/jgo.2019.30.e112.

93. Bahig H, Aubin F, Stagg J, et al. Phase I/II trial of durvalumab plus tremelimumab and stereotactic body radiotherapy for metastatic head and neck carcinoma. BMC Cancer. 2019. https://doi.org/10. 1186/s12885-019-5266-4.

94. Kelley RK, Abou-Alfa GK, Bendell JC, et al. Phase I/II study of durvalumab and tremelimumab in patients with unresectable hepatocellular carcinoma (HCC): phase I safety and efficacy analyses. J Clin Oncol. 2017;35:4073-4073. 